\title{
Evaluating the toxicity of the analgesic glutaminase inhibitor 6-diazo-5-oxo-l-norleucine in vitro and on rat dermal skin fibroblasts
}

\begin{abstract}
6-diazo-5-oxo-1-norleucine (DON) is a glutamine antagonist produced naturally by Streptomyces. It inhibits several glutamine-dependent enzyme pathways. Of particular note is its inhibitory effect on the mitochondrial enzyme, glutaminase (GLS), the primary producer of neuronal glutamate. Glutamate is an excitatory neurotransmitter released by primary sensory peripheral nerve terminals and spinal synaptic terminals during pain signaling. Previous work using the tail incision and inflammatory models of pain has demonstrated that a single application of the glutaminase inhibitor, DON, into a surgical incision or the paw of arthritic animals results in pain relief. Even though this compound shows promise as a therapeutic agent, limited data exist regarding its dermal toxicity. As a first approach, we evaluated the effect of several concentrations of DON, on the viability, mitochondrial oxidative capacity and proliferation of rat skin fibroblasts, and then examined the effect of DON after incubation with human liver microsomes on proliferation. Finally, we evaluated DON treated rat skin (tail and hind paw) for cellular necrosis, inflammation and mitotic bodies. No significant effects $(\mathrm{p}>0.05)$ of DON were noted on apoptosis, necrosis, and mitochondrial activity in experiments with cultured rat skin fibroblasts. Flow cytometry revealed the absence of apoptosis in cells treated at the $\mathrm{IC}_{50}$ of $232.5 \mu \mathrm{M}$. Enhanced toxicity post-exposure to human microsomes was not observed when compared to DON alone. The H\&E staining of the rat skin revealed no obvious pathology in the DON treatment group $(10 \mathrm{mM})$. DON has no/minimal cellular toxicity in vitro on dermal fibroblasts at concentrations that effectively provide analgesia. The local application of concentrations greater than the in vitro $\mathrm{IC}_{50}$ for $\mathrm{DON}$ revealed no in vivo skin toxicity. These data provide results indicating zero-to-minimal cellular toxicity with DON and support the further investigation of DON as an analgesic.
\end{abstract}

Keywords: glutaminase, glutamate, incision, post-surgical pain, vglut2, drg, spraguedawley rats, gls, don, 6-diazo-5-oxo-l-norleucine
Volume I Issue I - 2015

\section{Heith A Crosby,' Michael Ihnat, ${ }^{2}$ Kenneth E Miller'}

'Department of Anatomy and Cell Biology, Oklahoma State University-Center for Health Sciences, USA

2Department of Pharmaceutical Sciences, University of

Oklahoma-Health Sciences Center, USA

Correspondence: Heith A Crosby, Department of Anatomy and Cell Biology, Oklahoma State University-Center for Health Sciences, I I I West 17th Street,Tulsa, USA, Tel 91856I58I7, Email heith.crosby@okstate.edu

Received: February 24, 2015 | Published: March 25, 2015
Abbreviations: AIA, adjuvant-induced arthritis; BUN, blood urea nitrogen; CGRP, calcitonin gene-related peptide; CDK, cyclin dependent kinases; DON, 6-diazo-5-oxo-1-norleucine; EAA-R, excitatory amino acid receptor; EKG, electrocardiogram; FBS, fetal bovine serum; GI, gastrointestinal; GLS, glutaminase; $\mathrm{IC}_{50}$, inhibitory concentration $50 \%$; IV, intravenous; $\mathrm{LD}_{50}$, lethal dose $50 \%$; MTS, (3-(4,5-dimethylthiazol-2-yl)-5-(3-carboxymethoxyphenyl)2-(4-sulfophenyl)-2h-tetrazolium); MTT，3-(4,5-dimethylthiazol2-yl)-2,5-diphenyltetrazolium bromide; NADPH, nicotinamide adenine dinucleotide phosphate; PBS, phosphate buffered saline; PS, phosphatidylserine; PI, propidium iodide; SP, substance p; TSI, tail surgical incision

\section{Introduction}

Pain is a ubiquitous sign and symptom that affects the lives of an untold number of persons worldwide. Its description and effect has been the subject of countless writings over history. It was considered by Celsus (30BC-38AD) as one of the cardinal signs of acute inflammation. ${ }^{1}$ Currently, 100million Americans suffer from chronic pain and an estimated 76.2million Americans suffer from acute pain. ${ }^{2}$ The current estimate for the annual cost to treat chronic pain alone is $\$ 100$ billion. ${ }^{3}$ The impact on quality of life and physical activity is significant, with an estimated $42 \%$ of the affected population experiencing limitations in daily activities. ${ }^{4}$ Hyperalgesia is the exaggerated response to a noxious stimuli and allodynia is the nociceptive response to a non-noxious stimulus. ${ }^{5}$ Both responses are characteristic of tissue injury, such as a surgical wound. Primary sensory neurons innervating the skin are glutamatergic and, upon activation, release glutamate. ${ }^{6}$ Glutamate stimulates excitatory amino acid receptors (EAA-R) on primary afferents and this stimulation results in sensitization and activation of nearby primary afferents, enhancing the nociceptive sensation. During the inflammatory process, peripheral afferent terminals release glutamate and neuropeptides such as calcitonin gene-related peptide (CGRP) and substance P (SP). These neurons also release glutamate from synaptic terminals in the dorsal horn of the spinal cord, resulting in stimulation of second order neurons and furthering the transmission of the nociceptive stimuli. ${ }^{7-9}$ The primary producer of neuronal glutamate is the enzyme glutaminase (GLS) and GLS levels increase in the cell bodies of primary afferent sensory neurons in the adjuvant-induced arthritis (AIA) and tail surgical incision (TSI) models in the rat. ${ }^{10,11}$ Subsequently, increases in GLS are found in the sensory nerve terminals in the skin due to transport via peripheral nerve axons. ${ }^{10,11}$ This elevation of GLS allows sensory nerves in the skin to produce and release large amounts of glutamate during inflammation. Elevated glutamate release from sensory nerves contributes, in part, to the enhanced pain sensitivities (hyperalgesia and allodynia) of inflammation. ${ }^{6,7,10,12}$ Since GLS is responsible for the neuronal production of glutamate from glutamine, ${ }^{13,14}$ it is a potential therapeutic target for alleviating 
pain. Indeed, several classes of GLS inhibitors are effective analgesics when applied to peripheral sensory nerve terminals during AIA. ${ }^{10}$ A single intradermal injection of 6-diazo-5-oxo-1-norleucine (DON), a GLS inhibitor, into the inflammation site is capable of producing pain relief for several days..$^{10}$ GLS in peripheral nerve terminals, therefore, presents as a novel therapeutic target for both the treatment of acute and chronic pain.

DON is a glutamine analog that inhibits GLS by covalently binding to the glutamine receptor site. ${ }^{15,16}$ DON has been tested as a chemotherapeutic agent, however toxicity limited its approval and use in humans. ${ }^{17}$ Even though previous studies have defined DON's toxicity, this has been in the context of a chemotherapeutic agent. It is important to note that in analgesia studies DON is given as a one-time administration using a dose much smaller than the doses mentioned in previous preclinical toxicity studies and clinical chemotherapy regimens. The qualities of a single local application of a much smaller dose providing analgesia for several days allows for the possibility of safe use in addition to proven efficacy. One of the next steps in evaluating DON, as a locally applied analgesic, is to examine possible cytotoxic effects on skin cell types. In the current study, we examined the in vitro effect of various concentrations of DON and its microsomal metabolites on the rat skin fibroblast cell line, CRL1213 (ATCC), as it relates to cell proliferation, mitochondrial enzymatic activity, cell cycle, and apoptosis. Furthermore, we evaluated if microsomal activation of DON enhances its toxicity on $3 \mathrm{~T} 3$ fibroblasts and initiated a preliminary study of DON's toxicity in vivo in rat tail and hind paw skin cells for skin cellular necrosis, inflammation and mitotic bodies.

\section{Materials and methods}

\section{Cell culture}

Rat skin fibroblast FR (ATCC $\left({ }^{\circ}\right.$ CRL1213 ${ }^{\mathrm{TM}}$ ) used in these experiments were cultured in complete growth medium containing Eagle's Minimum Essential Medium (ATCC $($ ), and $10 \%$ fetal bovine serum (FBS) at $37^{\circ} \mathrm{C}$ in an atmosphere of $5 \% \mathrm{CO}_{2}$ and $95 \%$ air. This cell line was used in all experiments except the microsomal assay. Mouse embryonic fibroblast cells (NIH/3T3 ATCC ${ }^{\circledR}$ CRL-1658 ${ }^{\mathrm{TM}}$ ) used in these experiments were cultured in the complete growth

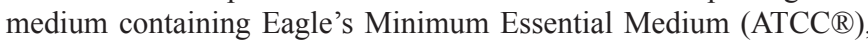
and $10 \%$ fetal bovine serum (FBS) at $37^{\circ} \mathrm{C}$ in an atmosphere of $5 \%$ $\mathrm{CO}_{2}$ and $95 \%$ air. This cell line was used in the microsomal assay.

\section{Annexin V I propidium iodide assay}

Rat skin fibroblasts FR (ATCC ${ }^{\circledR}$ CRL1213 ${ }^{\mathrm{TM}}$ ) were seeded in 96well plates at $1 \times 10^{5}$ cells $/ \mathrm{mL}$ and allowed to reach log phase growth. On day four, complete growth media was aspirated. In initial studies, $100 \mu \mathrm{l}$ of one of the following treatments were added: $0,0.00002$, $0.0002,0.002,0.02,0.2,2,20,200$ or $500 \mu \mathrm{M}$ concentrations of DON (Sigma-Aldrich) or the positive control $6 \mu \mathrm{M}$ camptothecin (SigmaAldrich) in OptiMem (Invitrogen) with 3\% FBS. Following a one, two, four or six-hour incubation, the treatments were aspirated and the cells were assessed for differences in apoptosis and cell viability. For later studies, $100 \mu 1$ of the following treatments were added: $0,2,20$, or $200 \mu \mathrm{M}$ concentrations of DON (Sigma-Aldrich) and the positive control $6 \mu \mathrm{M}$ camptothecin (Sigma-Aldrich) in OptiMem (Invitrogen) $3 \%$ FBS. Following forty-eight hours incubation, the treatments were aspirated and the cells were assessed for differences in apoptosis and cell viability. The Annexin V FITC assay kit (Cayman Chemical 600300) was used to detect apoptosis and cell viability. Absorbance and fluorescence were read at $485 \mathrm{~nm}$ excitation $/ 535 \mathrm{~nm}$ emission. Results were obtained using a Multimode Detector DTX 880 (Beckman Coulter).

\section{MTT assay}

Clear 96-well plates were seeded with FR (ATCC ${ }^{\circledR}$ CRL1213 ${ }^{\mathrm{TM}}$ ) rat fibroblasts at $2 \times 10^{4}$ cells $/ \mathrm{ml}$. On day 4 , complete growth media was aspirated, and $100 \mu \mathrm{l}$ of the following treatments were added: $0,2,20$, or $200 \mu \mathrm{M}$ concentrations of 6-diazo-5-oxo-1-norleucine and $6 \mu$ Mcamptothecin in OptiMem (Invitrogen) 3\% FBS. After 48 hours incubation, the treatments were aspirated and $10 \mu \mathrm{L}$ of 3-(4,5-dimethylthiazol-2-yl)-2,5-diphenyltetrazolium bromide (MTT) was added to each well. The plates were incubated for 2 hours at $37^{\circ} \mathrm{C}$, $95 \%$ air and $5 \% \mathrm{CO}_{2}$, until purple dye was visible. Detergent (sodium dodecyl sulfate) $100 \mu \mathrm{L}$ was added to each well and the plates were covered in aluminum foil and incubated in a dark area for 2 hours at room temperature. Absorbance and fluorescence were read at $485 \mathrm{~nm}$ excitation $/ 535 \mathrm{~nm}$ emission. Results were obtained using a Multimode Detector DTX 880 (Beckman Coulter).

\section{MTS assay}

Ninety-six well plates were seeded at 3000 cells/well with FR (ATCC® CRL1213 ${ }^{\mathrm{TM}}$ ) rat fibroblasts. On day 4, complete growth media was aspirated, and $100 \mu \mathrm{l}$ of the following treatments were added: $0,0.02,0.05,0.15,0.46,1.4,4,12,37,111,333$, or $1000 \mu \mathrm{M}$ concentrations of 6-diazo-5-oxo-L-norleucine- in OptiMem (Invitrogen) 3\% FBS. After 48 hours incubation, $20 \mu \mathrm{L}$ of MTS (3-(4,5-dimethylthiazol-2-yl)-5-(3-carboxymethoxyphenyl)-2-(4sulfophenyl)-2H-tetrazolium) (Promega) was added to each well. The plates were covered in aluminum foil and incubated for 2 hours at $37^{\circ} \mathrm{C}, 95 \%$ air, and $5 \% \mathrm{CO}_{2}$, until purple dye was visible. Absorbance and fluorescence were read at $495 \mathrm{~nm}$ excitation $/ 535 \mathrm{~nm}$ emission using a Synergy plate reader (Biotek).

\section{CyQUANT ${ }^{\circledR}$ assay}

Cell proliferation was analyzed using CyQUANT ${ }^{\circledR}$ Cell Proliferation Assay (Molecular Probes). Rat fibroblasts FR (ATCC ${ }^{\circledR}$ CRL1213 ${ }^{\mathrm{TM}}$ ) were plated at a density of 3,000 cells per well into white 96-well plates with clear bottoms and grown for 24 hours to allow for complete attachment. The cells were exposed to $100 \mu$ of one of the following concentrations of 6-diazo-5-oxo-L-norleucine- in OptiMem (Invitrogen): $0.10,0.45,1.30,4.10,12.30,37,111,333$ or $1000 \mu \mathrm{M}$ for 4 hours. Serum (10\% Cosmic calf, Hyclone, Logan UT) was added to the wells and the cells incubated for 44 hours at $37^{\circ} \mathrm{C}$. Exposure media was removed and the cells were lysed by freezing at $-80^{\circ} \mathrm{C}$ for 72 hours. The cells were thawed and exposed to a 1:400 dilution of CyQUANT $® / l y s i s$ buffer for 60 minutes. Plate fluorescence $(480 \mathrm{~nm}$ excitation, $520 \mathrm{~nm}$ emission) was read on a Synergy plate reader (BioTek).

\section{Flow cytometry}

Rat fibroblasts FR (ATCC ${ }^{\circledR}$ CRL1213 ${ }^{\mathrm{TM}}$ ) were plated at 30\% confluency in 6 well plates and allowed to attach overnight. DON was added at the $\mathrm{IC}_{50}$ concentration of $232.5 \mu \mathrm{M}$ as determined by the CyQUANT ${ }^{\circledR}$ Cell Proliferation Assay (Molecular Probes) for 2 hours in serum free media and then $10 \%$ Cosmic Calf ${ }^{\mathrm{TM}}$ Serum (Thermo Hyclone) was added. Cells were incubated at $37^{\circ} \mathrm{C}$ with $5 \% \mathrm{CO}_{2}$. After 48 hours incubation, cells were fixed in $50 \%$ ethyl alcohol solution and stored at $4^{\circ} \mathrm{C}$ for up to a week. After fixation, pelleted cells were suspended and labeled with propidium iodide 
$(25 \mu \mathrm{g} / \mathrm{mL})$ in phosphate buffered saline (PBS), $\mathrm{pH} 7.4$, containing $0.1 \%$ Triton X-100, $1 \mathrm{mg} / \mathrm{ml}$ sodium citrate, and $100 \mu \mathrm{g} / \mathrm{mL}$ RNase A. Cells were incubated for 30 minutes at $37^{\circ} \mathrm{C}$. Cells were subjected to flow cytometry using a side scatter plot versus fluorescence $(535 \mathrm{~nm}$ excitation $/ 617 \mathrm{~nm}$ emission).

\section{Microsomal toxicity}

Using a NIH-3T3 cell line, the following treatment groups were analyzed; DON and no microsomes, DON with microsomes, and untreated cells. Two $30 \mu \mathrm{L}$ aliquots of pooled human liver microsomes (Invitrogen, Grand Island, NY) were thawed on ice. Nicotinamide adenine dinucleotide phosphate (NADPH) $(10 \mathrm{mM})$ was added to prewarmed $\left(37^{\circ} \mathrm{C}\right) 100 \mathrm{mM}$ phosphate buffer $(\mathrm{pH} 7.0)$ and gently mixed. In two microcentrifuge tubes, $100 \mathrm{mM}$ sodium phosphate buffer, $10 \mathrm{mM}$ NADPH and $33.3 \mathrm{mM}$ DON were added into the tubes. In a third microcentrifuge tube, only $100 \mathrm{mM}$ sodium phosphate buffer and $10 \mathrm{mM}$ NADPH were added into the tube. Tubes were pre- incubated for five minutes at $37^{\circ} \mathrm{C}$. Microsomes were added to each of the tubes, vortexed briefly and incubated for 20 minutes at $37^{\circ} \mathrm{C}$. After the incubation period, the tubes were centrifuged $(13,000$ RPM) for five minutes at room temperature. The supernatant was added to each of four wells in a 96-well place. The supernatant was geometrically diluted in a 1:3 concentration five times in Opti-MEM to generate the dose-response curve (see CyQuant assay for specific incubation times and reagents). $\mathrm{IC}_{50}$ values were calculated using nonlinear regression dose-response analysis (variable slope) with Prism 6.x Software (Graphpad, San Diego CA).

\section{Animals}

HarlanSprague-Dawley rats $(\mathrm{n}=12$; male and female, Oklahoma State University Center for Health Sciences breeding colony originating from Charles River) were housed on a 12-hour light: 12hour dark cycle and given free access to food and water. Procedures were conducted according to guidelines from the National Institutes of Health ${ }^{18}$ and were approved by the Oklahoma State University Center for Health Sciences Institutional Animal Care and Use Committee. All appropriate efforts were made to minimize the number of animals used in these studies.

\section{DON injections}

HarlanSprague-Dawley rats, weighing between 250-350grams, were anesthetized with $5 \%$ isoflurane and oxygen $(3 \mathrm{~L} / \mathrm{min})$, in a plastic induction chamber, until mobility ceased. A nose cone was utilized to deliver maintenance anesthesia (isoflurane $2-3 \%, \mathrm{O}_{2} 1.5 \mathrm{~L} / \mathrm{min}$ ). Tails were measured to determine the midpoint of the proximal third of the tail. The proximal tails and right hind paws of the rats were cleaned using povidone iodine for disinfection. Control animals were naïve rats that received $50 \mu 1(0.05 \mathrm{ml})$ sterile saline $(0.9 \%)$ into the tails and right hind paws. The experimental animals received $50 \mu 1(0.05 \mathrm{ml})$ DON $(10 \mathrm{mM})$ injections made into the midpoint of the proximal portion of the tail and into the plantar aspect of the right hind paw. At 24 hours post-injection, animals were killed via $\mathrm{CO}_{2}$ asphyxiation and decapitation. Tissue was dissected from the area of injection (tail and right hind paw) and placed in 10\% neutral buffered formalin, $\mathrm{pH} 7.4$, for preservation. Samples were processed by Precision Histology Lab Inc. (Oklahoma City, Oklahoma) for hematoxylin eosin staining of paraffin embedded sections.

\section{Hematoxylin eosin stain}

Sections were deparaffinized 3 times with xylene, rehydrated in a descending series of ethanols (absolute-80\%), and washed in distilled water. Tissue sections were stained in Harris hematoxylin solution and rinsed with tap water three times. Staining was differentiated with $1 \%$ hydrochloric acid, rinsed with water three times, bluing in $1 \%$ ammonium hydroxide and counterstained in eosin. Sections were dehydrated with an ascending series of ethanols (70\%-absolute), and cleared in xylenes four times. Coverslips were apposed with Permount. A pathologist evaluated tissue (blinded samples) for apoptosis, cellular necrosis and inflammation.

\section{Statistics}

One-way ANOVA with Holm-Sidak post-hoc test was used to test for significance for the Annexin V, Propidium iodide, MTT and MTS assays. Analysis of DNA content was performed using MODFIT Lt software to generate percent $\mathrm{G}_{1} / \mathrm{G}_{0}$ phase, $\mathrm{S}$, and $\mathrm{G}_{2} / \mathrm{M}$ phase. Data were graphed as mean \pm standard error of the mean (sem). The $\mathrm{IC}_{50}$ values were determined using the CyQUANT ${ }^{\circledR}$ Cell Proliferation Assay. Comparison between group means was performed with either the Student's t-test ( 2 groups) or ANOVA ( $>2$ groups) to determine differences between experimental and control groups.

\section{Results}

\section{Apoptosis}

An early feature of apoptosis is a structural change in the plasma membrane, which involves the translocation of the membrane phospholipid, phosphatidylserine (PS), from the internal to the external layer of the cell membrane. Annexin $\mathrm{V}$ in the presence of calcium ions binds to PS and thus provides a very sensitive method for detecting apoptosis. It is important to note that the preliminary studies examined shorter exposure times, (i.e. 1, 2, 4, and 6 hours) to 6-diazo-5-oxo-1-norleucine (DON) and none revealed any significant changes in apoptosis or necrosis. This directed further experiments toward longer continuous exposure times (48 hours). The results of the Annexin V study (Figure 1) showed that media only wells had a fluorescence of $7369.3 \pm 769.62$ relative units $(\mathrm{ru})$. The relative fluorescence for media with cells was $9424.2 \pm 138.5$ ru, whereas fluorescence from cells treated with camptothecin $6 \mu \mathrm{M}$ (apoptotic cells) was $10356.7 \pm 175.4 \mathrm{ru}$. The fluorescence for cells exposed to either 2, 20, or $200 \mu \mathrm{M}$ DON was $9483.2 \pm 158.4 \mathrm{ru}, 9669.3 \pm 225.3 \mathrm{ru}$, $9524.7 \pm 169.8 \mathrm{ru}$, respectively. ${ }^{19}$ The only statistical significance $(\mathrm{p}<0.05)$ noted was the positive control, camptothecin.

\section{Rat Dermal Fibroblasts 48h Exposure DON Annexin}

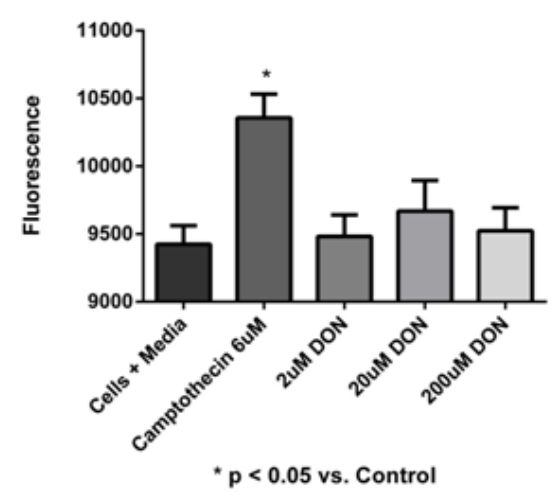

Figure I Annexin $\mathrm{V}$ assay. Rat dermal fibroblast cells exposed to DON concentrations of 2, 20, and $200 \mu \mathrm{M}$ for 48 hours continuous exposure. Media with cells $9424.20 \pm 138.5 \mathrm{ru}$; cells with camptothecin $(6 \mu \mathrm{M})$ I0356.7 $\pm 175.4 \mathrm{ru}$; $2 \mu \mathrm{M}$ DON 9483.2 $\pm 158.4 \mathrm{ru} ; 20 \mu \mathrm{M}$ DON 9669.3 $\pm 225.3 \mathrm{ru} ; 200 \mu \mathrm{M}$ DON $9524.7 \pm 169.8 \mathrm{ru}$. No significant difference was noted except for the positive control camptothecin ( ${ }^{*} \mathrm{p}<0.05 \mathrm{vs}$. control). Fluorescence were read at $485 \mathrm{~nm}$ excitation $/ 535 \mathrm{~nm}$ emission. Data reported as mean \pm sem. $\mathrm{N}=7$ per group. 


\section{Necrosis}

During the process of necrosis, the cell plasma membrane becomes compromised. Propidium iodide (PI) is a fluorescent compound that is excluded from viable cells and can only enter cells with a damaged plasma membrane. Once inside a cell, PI intercalates between DNA/ RNA bases and thus provides a very sensitive method for detecting necrosis. The results of the PI study showed that media only wells had a fluorescence of $2739.6 \pm 83.7 \mathrm{ru}$, whereas with the fluorescence of cells in media increased to $4058.8 \pm 180.2 \mathrm{ru}$. Camptothecin $(6 \mu \mathrm{M})$ treated cells showed a decrease in fluorescence to $3686.6 \pm 116.1 \mathrm{ru}$. The fluorescence for cells in 2, 20, and $200 \mu \mathrm{M}$, DON increased to $4522.1 \pm 155.3 \mathrm{ru}, 4502.0 \pm 171.1 \mathrm{ru}, 4208.7 \pm 169.4 \mathrm{ru}$, respectively. No statistical differences were observed (Figure 2). ${ }^{20}$

\section{Rat Dermal Fibroblasts 48h Exposure DON Propidium lodide}

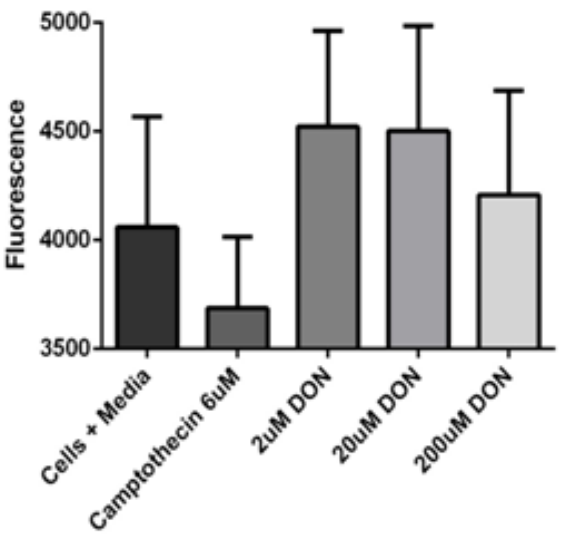

Figure 2 Propidium iodide relative fluorescence assay. Rat dermal fibroblast cells were exposed to DON concentrations of 2,20 , and $200 \mu \mathrm{M}$ or $6 \mu \mathrm{M}$ camptothecin for 48 hours. Cells in media $4058.8 \pm 180.2$ ru; camptothecin $(6 \mu \mathrm{M})$

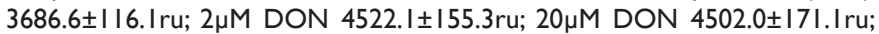
$200 \mu \mathrm{M}$ DON $4208.7 \pm 169.4 \mathrm{ru}$. No significant differences were noted. Fluorescence were read at $485 \mathrm{~nm}$ excitation $/ 535 \mathrm{~nm}$ emission. Data reported as mean \pm sem. $\mathrm{N}=7$ per group.

\section{Mitochondrial activity}

The MTT (3-[4,5-dimethylthiazol-2-yl]-2,5 diphenyltetrazolium bromide) and MTS (3-(4,5-dimethylthiazol-2-yl)-5-(3carboxymethoxyphenyl)-2-(4-sulfophenyl)-2H-tetrazolium) assays are based on the conversion of MTT/MTS into formazan crystals within the mitochondria by living cells. For most cell populations, the total mitochondrial activity is related to the number of viable cells. These assays can be used to measure the in vitro cytotoxic effects of drugs. The results of the MTT assay showed that media only wells had an absorbance of $0.37 \pm 0.08$, whereas cells in media had an absorbance that increased to $0.69 \pm 0.02$. Camptothecin $(6 \mu \mathrm{M})$ treated cells had an absorbance of $0.64 \pm 0.05$. Absorbance for cells treated in 2, 20, and $200 \mu \mathrm{M}$ DON increased to $0.68 \pm 0.06,0.64 \pm 0.02$, and $0.70 \pm 0.10$, respectively. No statistical differences were observed (Figure 3) ${ }^{21}$ In the MTS (3-(4,5-dimethylthiazol-2-yl)-5-(3-carboxymethoxyphenyl)2-(4-sulfophenyl)-2H-tetrazolium) assay, $0.001 \mu \mathrm{M}$ DON was set as an absorbance percentage of $100.00 \%$. No statistical significance was noted with percentages ranging from $87.9 \%(0.015 \mu \mathrm{M}$ DON) to $130.0 \%(1000.0 \mu \mathrm{M}$ DON) (Figure 4).

\section{Rat Dermal Fibroblasts 48h Exposure DON MTT Assay}

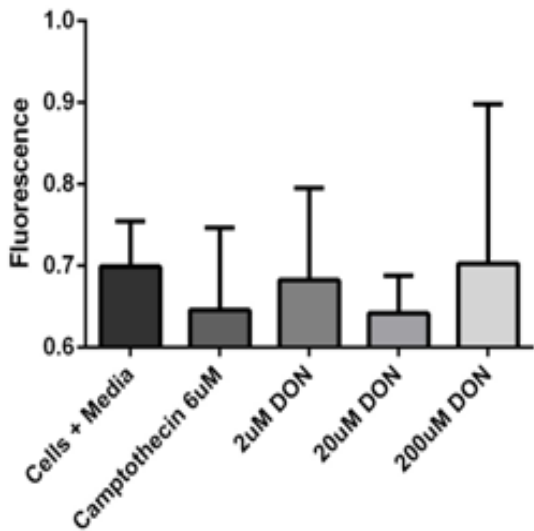

Figure 3 MTT assay. Rat dermal fibroblast cells were exposed to DON concentrations of 2,20 , and $200 \mu \mathrm{M}$ and $6 \mu \mathrm{M}$ camptothecin for 48 hours. MTT was added to the cells and the absorbance was read. MTT is used to determine mitochondrial activity. Cells in media $0.69 \pm 0.03$; camptothecin $(6 \mu \mathrm{M})$ treated cells $0.64 \pm 0.05 ; 2 \mu \mathrm{M}$ DON $0.68 \pm 0.06 ; 20 \mu \mathrm{M}$ DON $0.64 \pm 0.02$; $200 \mu$ M DON 0.70 \pm 0.10 . No significant differences noted. Data reported as mean \pm sem. Fluorescence were read at $485 \mathrm{~nm}$ excitation $/ 535 \mathrm{~nm}$ emission. $\mathrm{N}=7$ per group.

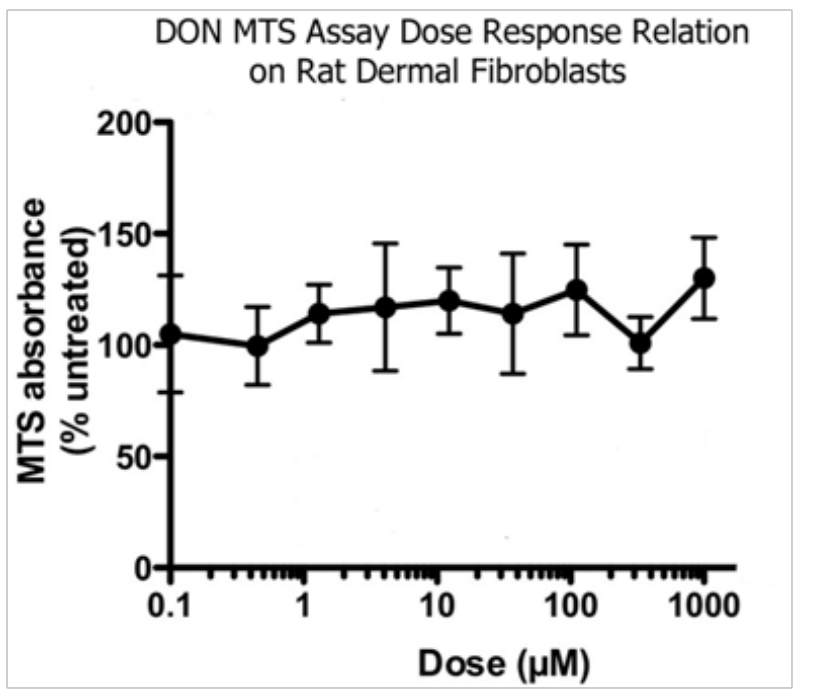

Figure 4 MTS assay. Rat dermal fibroblast cells were exposed to DON concentrations of $0,0.02,0.05,0.15,0.46,1.4,4,12,37,111,333$, and $1000 \mu \mathrm{M}$ for 48 hours. DON $0.001 \mu \mathrm{M}$ was set as an absorbance percentage of $100.00 \%$. No statistical significance was noted with percentages ranging from $87.9 \%$ $(0.015 \mu \mathrm{M} D O N)$ to $130.0 \%(1000.0 \mu \mathrm{M}$ DON). Absorbance were read at $485 \mathrm{~nm}$ excitation $/ 535 \mathrm{~nm}$ emission. Data reported as mean \pm sem. $\mathrm{N}=4$ per group.

\section{Cell proliferation}

Cell proliferation was analyzed using the CyQUANT ${ }^{\circledR}$ assay. This is a sensitive, fluorescence-based assay for determining cell number from cultures. The CyQUANT ${ }^{\circledR}$ GR fluorescent dye binds to DNA and can be measured using excitation and emission wavelengths. The fluorescence emission of the bound dye correlates linearly with cell number over a large range (i.e., $50-50,000$ cells per $200 \mu 1) .{ }^{22}$ 
The CyQUANT ${ }^{\circledR}$ absorbance percentage was set at $100 \%$ for the rat fibroblast cells treated with $0.10 \mu \mathrm{M}$ DON. No statistical differences were observed from cells treated with $0.45-10 \mu \mathrm{M}$ DON. At $30 \mu \mathrm{M}$ DON, CyQUANT ${ }^{\circledR}$ absorbance was reduced to $77.1 \%$. Further reductions (60.0-51.3\%) in CyQUANT ${ }^{\circledR}$ absorbance occurred at $37-333 \mu \mathrm{M}$ DON. The greatest reduction $(38.2 \%)$ in CyQUANT ${ }^{\circledR}$ absorbance occurred at $1000 \mu \mathrm{M}$ DON. In the cell proliferation assay, the half maximal inhibitory concentration $\left(\mathrm{IC}_{50}\right)$ for 6-diazo-5-oxo1-norleucine (DON) on rat dermal fibroblasts was determined to be $232.5 \mu \mathrm{M}$ (Figure 5).

\section{Cell cycle}

Utilizing flow cytometry, the population of cell replication states can be analyzed. This occurs by treating cells with a nucleic acid intercalating fluorescent dye, then analyzing the fluorescence properties of each cell in the population. Quiescent and G1 cells have one copy of DNA and will have one times (1X) the fluorescence intensity. Cells in G2/M phase of the cell cycle have two copies of DNA and have two times (2X) the intensity. Cells in S phase are synthesizing DNA and have fluorescence values between the $1 \mathrm{X}$ and $2 \mathrm{X}$ intensity. The flow cytometry control data for the $\mathrm{G}_{1}$ phase was $69.5 \%$; the $\mathrm{G}_{2} / \mathrm{M}$ phases were $15.1 \%$, and the $\mathrm{S}$ phase $15.3 \%$. Cells incubated in DON $(232.5 \mu \mathrm{M})$ for 24 hour were $71.0 \%$ in $\mathrm{G}_{1}$ phase, $5.8 \%$ for $\mathrm{G}_{2} / \mathrm{M}$ phases, and $\mathrm{S}$ phase at $23.2 \%$. At 48 hours of $\mathrm{DON}$ exposure, cells in $\mathrm{G}_{1}$ phase were $75.0 \%, 13.6 \%$ for $\mathrm{G}_{2} / \mathrm{M}$ phases, and $11.4 \%$ for $S$ phase ${ }^{23}$ For all groups no sub- $\mathrm{G}_{1}$ peak was observed indicating no apoptosis, in spite of longer exposure times to DON. Furthermore, cell cycle phase distribution is similar between controls and DON treated cells (Figure 6). These data support the previous annexin and propidium iodide experiments revealing no apoptosis or necrosis respectively.

Rat Dermal Fibroblasts Cyquant Assay

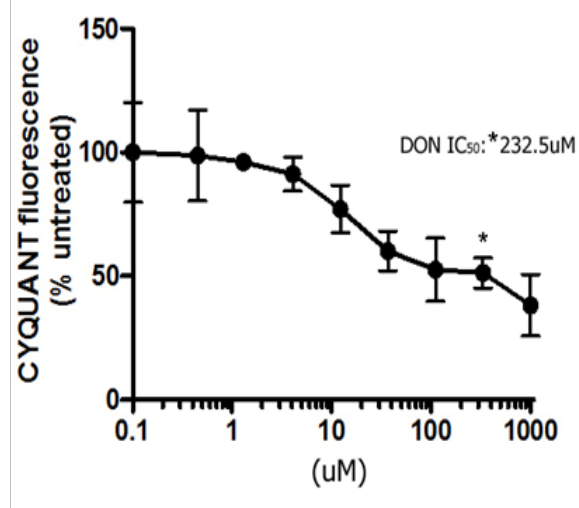

$* p<0.05$ vs. cells treated $0.45-10$ uM DON

Figure 5 Cell proliferation was analyzed using the CyQUANT ${ }^{\circ}$ assay. The CYQUANT® absorbance percentage was set at $100 \%$ for the rat fibroblast cells treated with $0.10 \mu \mathrm{M} \mathrm{DON}$. No statistical differences were observed from

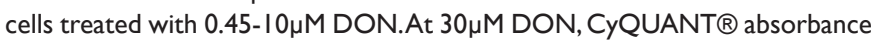
was reduced to $77.1 \%$. Further reductions $(60.0-51.3 \%)$ in CyQUANT® absorbance occurred at $37-333 \mu \mathrm{M}$ DON. The greatest reduction $(38.2 \%)$ in CyQUANT ${ }^{\circledR}$ absorbance occurred at $1000 \mu \mathrm{M}$ DON. The $\mathrm{IC}_{50}$ is $232.5 \mu \mathrm{M}$. $*_{p}<0.05$ vs cells treated with $0.45-10 \mu \mathrm{M}$ DON. Data reported as mean \pm sem. Fluorescence were read at $480 \mathrm{~nm}$ excitation $/ 520 \mathrm{~nm}$ emission. $\mathrm{N}=4$ per group.

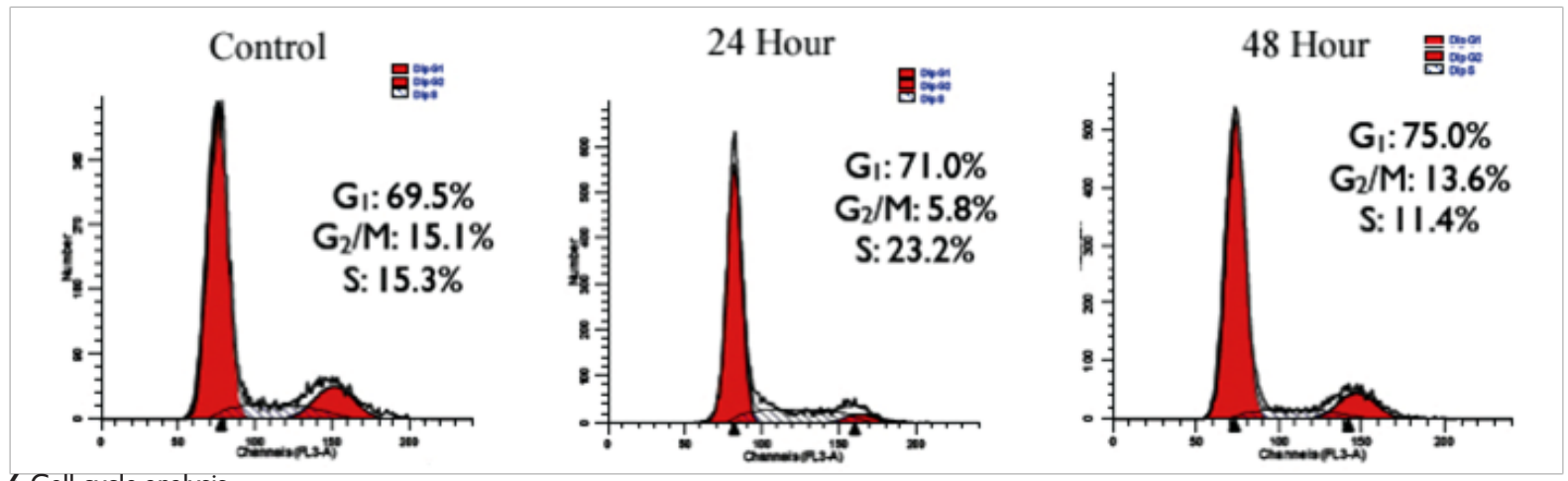

Figure 6 Cell cycle analysis.

Flow cytometric analysis of rat dermal fibroblast cells (ATCC CRL-I2I3) in the absence (control) and presence of $232.5 \mu \mathrm{M}$ DON for 24 and 48 hours. No Sub-G, peaks are evident after 24 and 48 hours of DON treatment indicating an absence of apoptosis.

\section{Cell proliferation microsomal metabolites}

PrestoBlue ${ }^{\circledR}$ (Life Technologies ${ }^{\mathrm{TM}}$ ) reagent is a resazurin-based solution that functions as a cell viability indicator by using the reducing power of living cells to quantitatively measure the proliferation of cells. The PrestoBlue ${ }^{\circledR}$ reagent contains a cell permeant compound that is blue in color and virtually non-fluorescent. When added to cells, the PrestoBlue ${ }^{\circledR}$ reagent is modified by the reducing environment of the viable cell and turns red in color and becomes highly fluorescent. This change can be detected using fluorescence or absorbance measurements. The absorbance percentage was set at $100 \%$ for the mouse embryonic fibroblast cells treated with $0.10 \mu \mathrm{M}$ DON. No statistical differences were observed from cells treated with up to $1000 \mu \mathrm{M} \mathrm{DON} .{ }^{24}$ In the cell proliferation assay, the half maximal inhibitory concentration $\left(\mathrm{IC}_{50}\right)$ for 6-Diazo-5-oxo-1-norleucine (DON) on mouse embryonic fibroblasts was determined to be $>1000 \mu \mathrm{M}$
(Figure 7) for the groups treated with and without human liver microsomes.

\section{Histologic findings hind paw and tail skin}

In naive control skin, epidermal and dermal cellular morphology had a normal appearance with an occasional apoptotic body or mitotic figure. In saline injected skin, occasional apoptotic bodies or mitotic figures were observed in the epidermis. In saline injected tail skin, one animal had an area of the epidermal keratin layer with a focal area of excoriation that was acutely inflamed. This may have been trauma (rubbing, scratching, etc.) rather than study related. In saline injected hind paw skin there was a focal area of the subcutis, containing a moderate focal influx of inflammatory cells that appeared to consist primarily of neutrophils and lymphoid cells. There also appeared to be evidence of mild tissue necrosis associated with the cellular infiltrate. 
In DON injected skin (tail, hind paw), there was no evidence of cellular necrosis or inflammation. In the epidermis of DON treated skin there were occasional mitotic figures and apoptotic bodies (Figure 8).

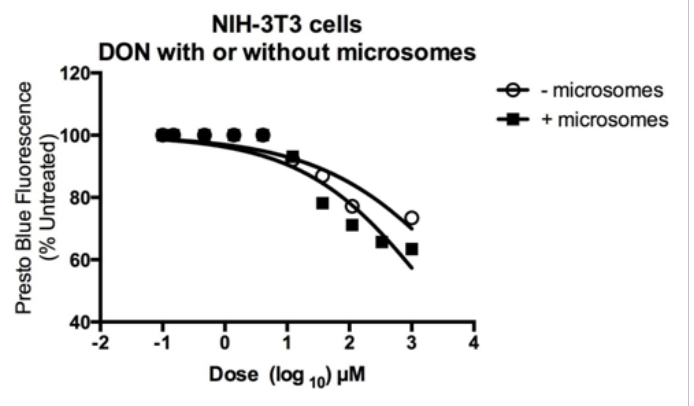

Figure 7 Microsomal cell Viability assay using PrestoBlue $®$. The absorbance percentage was set at $100 \%$ (excitation $570 \mathrm{~nm}$, emission $600 \mathrm{~nm}$ ) for the mouse embryonic fibroblast cells treated with $0.10 \mu \mathrm{M}$ DON. No statistical differences were observed from cells treated with up to $1000 \mu \mathrm{M} D O N$. The $I C_{50}$ for DON treated mouse embryonic fibroblasts was determined to be $>1000 \mu \mathrm{M}$ for both treatment groups (with and without microsomes). $\mathrm{N}=7$ per group.
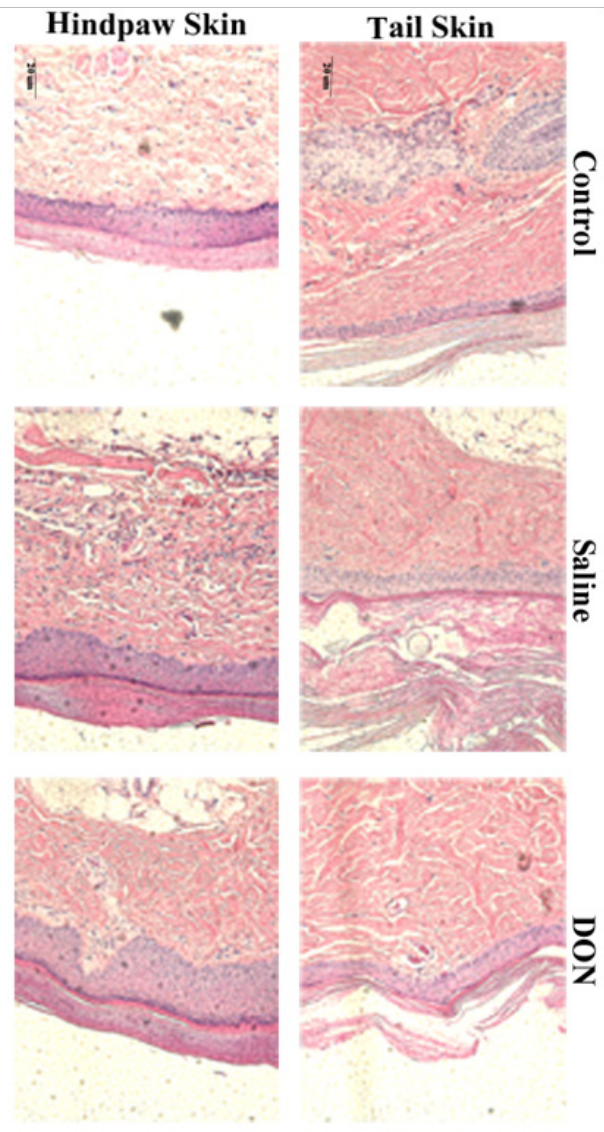

Figure 8 Skin toxicity.

Figure 8A Hind paw skin control.

Figure 8B Tail skin control.

Figure 8C Hind paw skin saline injection.

Figure 8D Tail skin saline injection.

Figure 8E Hind paw skin DON injection.

Figure 8F Tail skin DON.

\section{Discussion}

DON is a structural analog of L-glutamine and disrupts several glutamine dependent pathways, including purine and pyrimidine biosynthetic pathways, thereby resulting in varied side effects in vivo..$^{25}$ DON was initially investigated in clinical trials as early as 1957. Previous research in animal tumor models found DON effective for several types of cancers, (e.g., lung, mammary, colon). ${ }^{17}$ Preclinical studies examining the toxicity of the DON pro-drug, azotomycin, in murine models revealed a median lethal dose $\left(\mathrm{LD}_{50}\right)$ of $262 \mathrm{mg}$ / $\mathrm{kg}$ following a single intraperitoneal (ip) dose. When azotomycin is given in repeated daily ip doses the $\mathrm{LD}_{50}$ decreased to $0.86 \mathrm{mg} / \mathrm{kg}$, demonstrating a cumulative lethal effect, where large single doses are tolerated, small repeated doses proved lethal. ${ }^{26}$ In studies designed to investigate azotomycin-induced toxicity in relation to dosing schedule, three schedules were evaluated: single intravenous (IV) injection; single 24-hour IV infusion, and weekly IV doses for six weeks. These studies, performed in Beagle dogs, confirmed that the drug is best tolerated as a quick IV dose. In animals, the toxicity profiles were similar no matter the dosage regimen and are summarized below. Gastrointestinal (GI) toxicity, mainly noted by vomiting and anorexia, occurred at nearly all doses tested. At higher doses, GI bleeding and pathologic changes of the gastrointestinal epithelium were noted. It is important to note that large doses will result in GI toxicity with any regimen. Hematology toxicity increased as doses increased, as indicated by neutropenia, lymphocytopenia and thrombocytopenia. Hepatotoxicity was noted by increased transaminase and alkaline phosphatase levels. Increased blood urea nitrogen (BUN) levels, proteinuria, polydipsia and polyuria were suggestive of nephrotoxicity. Both heart and lung toxicities were found as evidenced by epicardial and endocardial hemorrhage and focal pulmonary fibrosis and mild focal chronic pneumonitis. High doses, $50-100 \mathrm{mg} / \mathrm{kg} /$ day IV, given daily for up to 7 weeks resulted in changes in the electrocardiogram (EKG). ${ }^{26}$

Phase I trials in humans of DON utilizing doses up to $500 \mathrm{mg} / \mathrm{m}^{2}$ showed nausea, vomiting, mucositis, and thrombocytopenia, as the major side effects, with nausea and vomiting being the dose limiting side effect. ${ }^{27}$ Phase II trials demonstrated less toxicity at doses of $50 \mathrm{mg} / \mathrm{m}^{2}$, which were exhibited mainly as mild leukopenia. In larger doses $\left(>50 \mathrm{mg} / \mathrm{m}^{2}\right)$, significant nausea and vomiting was noted with minimal efficacy. ${ }^{28,29}$ In human clinical trials, most patients received oral doses in the range of $0.2-1.1 \mathrm{mg} / \mathrm{kg} /$ day. Overall, gastrointestinal toxicity was most prevalent, with almost no myelotoxicity when DON was given alone in the low dosage range. However, it was reported that frequent administrations of DON increased its toxicity and by administering intermittently (every 4 days), higher total doses were better tolerated. ${ }^{28}$ In effect, these side effects prevented DON from becoming a viable chemotherapeutic. Although previous studies have defined DON's oral and intravenous toxicity, this has been in the context of a chemotherapeutic agent. When considered as an analgesic agent, studies have used either a local injection or topical application of a single dose much smaller than the doses in preclinical toxicity and clinical chemotherapy applications. ${ }^{10,11}$ These qualities of a single local application of a much smaller dose providing analgesia for several days allows for the possibility of safe and effective use in the appropriate clinical context.

We began our investigation by examining the in vitro effect of various concentrations of DON on the rat skin fibroblast cell line, CRL1213 (ATCC) as it relates to cell viability and apoptosis. These studies revealed no significant effects following continuous exposure with DON for 48 hours at concentrations comparable to the doses given in AIA studies. We then sought to examine DON's 
effect on mitochondrial function. Since GLS is found primarily in the mitochondria, we proposed to test for mitochondrial toxicity. Using the MTT and MTS assays DON demonstrated a non-significant toxic effect ( $p>0.05$ ) on rat skin fibroblasts exposed continuously for 48 hours with concentrations up to $1000 \mu \mathrm{M}$. As expected campothecin a proapoptotic agent showed significant increase $(p<0.05)$ in the apoptotic assay (Annexin V), and a non-significant effect $(\mathrm{p}>0.05)$ in all other assays.

Next we sought to determine the $\mathrm{IC}_{50}$ of $\mathrm{DON}$ on rat dermal fibroblasts. Our results showed a DON $\mathrm{IC}_{50}$ as determined using a CyQUANT ${ }^{\circledR}$ cell proliferation assay to be $232.5 \mu \mathrm{M}$ in the rat skin dermal fibroblast cell line CRL1213 (ATCC). Next we treated rat skin dermal fibroblast cells with the $\mathrm{IC}_{50}$ dose of $232.5 \mu \mathrm{M}$ to examine the effect on cell cycle. The flow cytometry data showed an increase in S phase from $11 \%$ to $23 \%$ at 24 hours compared to control however by 48 hours it returned back to $15 \%$. Important to note is that no evidence of apoptosis or necrosis was noted. The question arises if not apoptosis or necrosis, then what might cause the decrease in cell proliferation? Glutamine is the required nitrogen donor for nucleotide synthesis. Nucleotide synthesis is required for cell proliferation. As mentioned earlier, ${ }^{11}$ DON's main chemotherapeutic effect is blockade of purine (nucleotide) biosynthesis by inhibition of amidophosphoribosyltransferase (EC 2.4.2.14), phosphoribosylformylglycinamidinesynthase (EC 6.3.5.3), and a number of amidotransferases. This inhibition in nucleotide syntheis might possibly be the cause of decreased cellular proliferation. ${ }^{30}$ Other possible explanations on DON's effect on cell proliferation however not yet tested might include regulation of cyclin dependent kinases (CDKs), which are core activators of the cell cycle control system and function to turn specific proteins on and off at appropriate times in the cell cycle. Another possibility is regulation at the G1 checkpoint, which is the time when cells prepare for DNA replication. Furthermore, cells require growth factor-dependent signals up to a point in late G1, referred to as the "restriction point", after which the transition is made into $\mathrm{S}$ phase. This is a crucial control point that is often dysregulated in cancer. The transition between early G1 and late G1 ("start") is regulated by CDK's and therefore can be modulated by compounds acting as CDK inhibitors. Still another possibility is the activation of anti-mitogen pathways. An example is the TGFß-Smad anti-mitogenic pathway. TGF $\beta$ is an extracellular protein that binds a cell surface receptor and causes cells to arrest their cell cycle and enter G0. Upon binding to TGFß, the receptor phosphorylates proteins in the cytoplasm termed Smads. The Smad proteins enter the nucleus and function as transcription factors to turn on specific target genes. Thus, TGF $\beta$ arrests cell division by turning on transcription of the gene for a CDK inhibitor. ${ }^{31}$

The next step was to determine how liver metabolism might affect DON. In other words, does microsomal metabolism make DON less or more toxic? To address this question, we treated NIH 3T3 mouse fibroblast cells with DON that was incubated with human liver microsomes. The results revealed very high $\mathrm{IC}_{50}$ values $(>1000 \mu \mathrm{M}$ with microsomes and without microsomes). Whether microsomal activation does enhance the toxicity of DON is still not known, however concentrations $>1000 \mu \mathrm{M}$ did not reveal any toxicity. So whether microsomal activation occurs and has any biological significance will require further examination. The results of these experiments posed the question, why are there differences in $\mathrm{IC}_{50}$ values between the rat and mouse fibroblast cell lines? Possible considerations might include developmental differences in cell lines (i.e., adult versus embryonic) resulting in differences in intrinsic cell sensitivity. These variations could occur in membrane permeability, intracellular synthetic pathways, adaptive and recovery mechanisms, biochemical transformation of the compound, receptor and transporter binding. To conclude, all the $\mathrm{IC}_{50}$ values were large when compared to analgesic doses used in previous studies. This offers some margin of safety and provides encouraging results for continued toxicity testing of DON.

Our studies extended to the histological examination of DON on rat tail and hind paw skin. To compliment previous efficacy studies, a $0.05 \mathrm{ml}$ injection volume was used as in skin incision studies. ${ }^{11}$ Since the hind paw has been used in several AIA studies, hind paw skin was included in our analysis. We used a concentration of $10 \mathrm{mM}$ DON in this study for a dose equaling ten times the largest cytotoxicity $\mathrm{IC}_{50}$ $(>1000 \mu \mathrm{M})$. The histological examination of the epidermis showed very few apoptotic bodies or mitotic figures in the saline injected tail skin. In saline injected hind paw skin there was a focal area of the subcutis containing a moderate focal influx of inflammatory cells that appeared to consist primarily of neutrophils and lymphoid cells. There also appeared to be evidence of mild tissue necrosis associated with the cellular infiltrate. In DON injected skin (tail, hind paw), there was no evidence of cellular necrosis or inflammation, but the epidermis showed occasional mitotic figures and apoptotic bodies. If DON were toxic at the $10 \mathrm{mM}$ dose, we would expect to see an increase in the formation of apoptotic bodies and/or necrosis. Apoptotic body formation is a mechanism to prevent leakage of potentially toxic or immunogenic cellular contents of dying cells and minimize inflammation, autoimmune reactions and tissue destruction. ${ }^{32}$ Furthermore if DON were proapoptotic, we would expect an unusual number of cells undergoing cell division as indicated by increased numbers of mitotic figures or spindles. However few mitotic figures were observed. In total, these results provide encouraging data regarding the safety of locally applied DON at concentrations ten times the largest $\mathrm{IC}_{50}$ concentration $(>1000 \mu \mathrm{M})$. Only occasional apoptotic bodies and mitotic figures were noted, well within normal limits. In summary, the skin histology of DON injected tissue was similar to the majority of the controls showing no apparent cytotoxicity.

In these studies we sought to clarify, characterize and define the toxicological effect DON has on rat skin fibroblast cellular viability, apoptosis, necrosis, cell proliferation, cell cycle alteration, and skin cytotoxicity over a range of concentrations. Our preliminary studies examined shorter exposure times, (i.e., 1, 2, 4, and 6 hours) and no dose showed any significant changes in rat fibroblasts. This directed our experiments towards a longer continuous exposure time, (i.e., 48 hours). Most important to note is that cells treated continuously with DON over 2 orders of magnitude greater than the AIA analgesic dose showed no significant toxic effects on the early apoptotic marker, annexin $\mathrm{V}$, cell viability, mitochondrial oxidative capacity, cell cycle status, and skin morphology. It is important to emphasize that doses used in preclinical analgesic studies are much smaller ( $1 \mathrm{ml}$ of $2 \mathrm{mM}$ DON or less) when compared to chemotherapeutic use and administered locally. The lack of toxic effects is likely due to the small concentrations, volumes and localized application used in analgesia when compared to doses used in cancer chemotherapy studies. Furthermore, the local application of a concentration ten times greater than the largest $\mathrm{IC}_{50}$ observed in our studies and 5 times larger than the analgesic dose used in the incision model for pain revealed no skin toxicity. ${ }^{11}$ Overall, these results offer a first step to help characterize and define the effect DON and its microsomal metabolites have on rat skin fibroblast cellular viability, proliferation, mitochondrial enzymatic activity, apoptosis, skin toxicity over a range 
of concentrations. These data support the further investigation of DON as an analgesic. Additional in vivo studies are needed to evaluate the safety of acute and chronic DON administration in applications where it may be used clinically.

\section{Conclusion}

Our studies showed that DON has no/minimal cellular toxicity in rat dermal skin fibroblasts at concentrations that effectively provide analgesia. As mentioned previously, cell proliferation $\left(\mathrm{IC}_{50}\right)$ was affected at concentrations two-fold greater than analgesia doses in previous inflammatory pain experiments. Furthermore, the local application of DON at concentrations greater than ten times the $\mathrm{IC}_{50}$ for cell proliferation revealed no skin toxicity. It is important to note that the DON administrations in the tail surgical incision model were given as a single application, whereas in vitro cytotoxicity was observed with continuous DON exposure for 48hrs. Additionally, previous studies have demonstrated that DON injected in naive rats showed no alterations in mechanical or thermal sensitivity providing support for lack of functional toxicity in vivo. ${ }^{11}$ This also is true for hind paw injection and topical application. It is important to highlight the fact that in the analgesia studies it is only a single DON dose administered by local application. This is contrasted with the oral and parenteral routes of administration of much larger doses and repeated chemotherapeutic regimens, allowing for higher drug tissue concentrations. When taken in this perspective, further investigation of this and other glutaminase inhibitors for the local application in the treatment of pain are warranted.

\section{Acknowledgements}

Authors have no conflicts of interest. This work was supported in part by the University of Oklahoma College of Pharmacy, Oklahoma State University Center for Health Sciences Intramural Grant and the NIH Grant AR047410 (KM).

\section{Author contribution}

HC, KM, MI designed research, conducted research, analyzed data. $\mathrm{HC}$ and $\mathrm{KM}$ wrote the paper; $\mathrm{HC}$ had the primary responsibility for final content; all authors read and approved the final manuscript.

\section{Conflict of interest}

The author declares no conflict of interest.

\section{References}

1. Vogel, Wolfgang H, Berke, et al. Brief history of vision and ocular medicine. Amsterdam: Kugler/Wayenborgh; 2009. p. 97.

2. Pain management. National Institutes of Health; 2010.

3. Bradford HF, Ward HK, Foley P. Glutaminase inhibition and the release of neurotransmitter glutamate from synaptosomes. Brain Res. 1989;476(1):29-34.

4. Eksterowicz N. It's the decade of pain control and research: Are we really making a difference? 2004.

5. Woolf CJ. Somatic pain — pathogenesis and prevention. Br J Anaesth 1995;75(2):169-176.

6. Fein A. Nociceptors and the Perception of Pain. 2012

7. Lawand NB, Willis WD, Westlund KN. Excitatory amino acid receptor involvement in peripheral nociceptive transmission in rats. Eur $J$ Pharmacol. 1997;324(2-3):169-177.
8. Miller KE, Douglas VD, Kaneko T. Glutaminase immunoreactive neurons in the rat dorsal root ganglion contain calcitonin gene-related peptide (CGRP). Neurosci Lett. 1993;160(1):113-116.

9. Zhang Z, Wang T, Miller KE. Accumulation of glutaminase, VGluT2, and substance $P$ in sciatic nerve following adjuvant-induced arthritis in rat. Society for Neuroscience. 586:10.

10. Miller KE, Hoffman EM, Sutharshan M, et al. Glutamate pharmacology and metabolism in peripheral primary afferents: Physiological and pathophysiological mechanisms. Pharmacol Ther. 2011;130(3):283309.

11. Crosby HA. Evaluating the enzyme glutaminase in primary sensory afferents in a rat tail surgical incision model, and evaluating the analgesic effect in vivo and the in vitro toxicity of the glutaminase inhibitor 6-diazo-5-oxo-l-norleucine on rat dermal skin fibroblasts. Doctoral Dissertation. Oklahoma: University of Oklahoma Health Sciences Center; 2013. p. 1-171.

12. Bardoni R, Torsney C, Tong CK, et al. Presynaptic NMDA receptors modulate glutamate release from primary sensory neurons in rat spinal cord dorsal horn. J Neurosci. 2004;24(11):2774-2781.

13. Pfizer Medical Division. The burden of pain among adults in the United States. 2008.

14. Daikhin Y, Yudkoff M. Compartmentation of brain glutamate metabolism in neurons and glia. J Nutr. 2000;130(4S Suppl):1026S$1031 \mathrm{~S}$.

15. Shapiro RA, Clark VM, Curthoys NP. Inactivation of rat renal phosphate-dependent glutaminase with 6-diazo-5-oxo-L-norleucine. Evidence for interaction at the glutamine binding site. $J$ Biol Chem. 1979;254(8):2835-2838.

16. Hoffman EM, Edwards KM, Herzog BH, et al. Intrathecal glutaminase inhibition with 6-diazo-5-oxo-L-norleucine in rat dorsal root ganglion neurons. Proceedings of the Society for Neuroscience. $2006 ; 737.5$

17. Ovejera AA, Houchens DP, Catane R, et al. Efficacy of 6-diazo-5oxo-L-norleucine and $\mathrm{N}$-[N-gamma-glutamyl-6-diazo-5-oxonorleucinyl]-6-diazo-5-oxo-norleucine against experimental tumors in conventional and nude mice. Cancer Res. 1979;39(8):3220-3224.

18. National Research Council (NRC). Guidelines for the care and use of animals in neuroscience. Washington, USA; 2003. p. 1-223.

19. Scharstuhl A, Mutsaers HA, Pennings SW, et al. Curcumin-induced fibroblast apoptosis and in vitro wound contraction are regulated by antioxidants and heme oxygenase: implications for Scar Formation. $J$ Cell Mol Med. 2009;13(4):712-725.

20. Niu G, Chen X. Apoptosis imaging: beyond annexin V. J Nucl Med. 2010;51(11):1659-1662.

21. Waring P, Lambert D, Sjaarda A, et al. Increased cell surface exposure of phosphatidylserine on propidium iodide- negative thymocytes undergoing death by necrosis. Cell Death Differ. 1999;6(7):624-637.

22. Jones LJ, Gray M, Yue ST, et al. Sensitive determination of cell number using the CyQUANT ${ }^{\circledR}$ cell proliferation assay. J Immunol Methods. 2001;254(1-2):85-98.

23. Knutsen JH, Rein ID, Rothe C, et al. Cell-cycle analysis of fission yeast cells by flow cytometry. PLoS One. 2011;6(2):e17175.

24. Mohammadi-Bardbori A, Ghazi-Khansari M. Comparative measurement of cyanide and paraquat mitochondrial toxicity using two different mitochondrial toxicity assays. Toxicol Mech Methods. 2007;17(2):87-91.

25. Sklaroff RB, Casper ES, Magill GB, et al. Phase I study of 6-diazo-5oxo-L-norleucine (DON). Cancer Treat Rep. 1980;64(12):1247-1251. 
26. Clinical brouchure. DON (NSC) 7365. National Cancer Institute; 1979.

27. Kovach JS, Eagan RT, Powis G, et al. Phase I and pharmacokinetic studies of DON. Cancer Treat Rep. 1981;65(11-12):1031-1036.

28. Lynch G, Kemeny N, Casper E. Phase II evaluation of DON (6diazo-5-oxo-1-norleucine) in patients with advanced colorectal carcinoma. Am J Clin Oncol. 1982;5(5):541-543.

29. Magill GB, Myers WP, Reilly HC, et al. Pharmacological and initial therapeutic oberservations on 6-diazo-5-oxo-L-norleucine (DON) in human neoplastic disease. Cancer. 1957;10(6):1138-1150.
30. Ahluwalia GS, Grem JL, Hao Z, et al. Metabolism and action of amino acid analog anti-cancer agents. Pharmacol Ther. 1990;46(2):243-271.

31. Harashima H, Dissmeyer N, Schnittger A. Cell cycle control across the eukaryotic kingdom. Trends Cell Biol. 2013;23(7):345-356.

32. Elmore S. Apoptosis: A review of programmed cell death. Toxicol Pathol. 2007;35(4):495-516. 